\title{
Analysis of Accurate Marketing Strategy of Online Advertising in Big Data Era
}

\author{
Ying Jiaming ${ }^{1, \mathrm{a}^{*}}$ \\ ${ }^{1}$ School of business administration, Zhejiang Gongshang University, Hangzhou, Zhejiang, China \\ $a^{*} 869815325 @ q q . c o m$
}

\begin{abstract}
The 21 st century has become an era of "Internet + " and "data is king." Commercial advertising has gradually changed from traditional models to online advertising relying on big data. Using data collection, mining and sorting, companies can apply the analysis results to the marketing, thereby further improving the efficiency and accuracy of advertising. This article analyzes the current situation and problems of online advertising, and formulates accurate marketing strategies for online advertising from the perspective of big data technologies, user privacy protecting, advertising platforms, advertising formats, and advertising relevance.At last, this paper makes the conclusions and rethink on online advertising marketing to promote the prosperity and good development of China's online advertising.
\end{abstract}

Keywords: big data, online advertising, accurate marketing

\section{大数据时代网络广告精准营销策略分析}

应嘉明 $1, \mathrm{a}^{*}$

\author{
${ }^{1}$ 浙江工商大学工商管理学院, 杭州, 浙江, 中国 \\ a*869815325@qq.com
}

\section{摘要}

21 世纪已经成为了 “互联网+”和 “数据为王” 的时代, 商业广告从依靠传统模式逐渐转变为依靠大 数据技术的网络广告。通过数据收集、挖掘以及整理等过程, 企业将分析结果恰当地运用到商业活动 营销中，从而进一步提高广告的效率和精准度。本文通过分析网络广告的现状以及面临的问题，针对 大数据技术、用户隐私保护、广告平台、广告形式以及广告关联等方面制定大数据时代网络广告的精 准营销策略，并在最后对网络广告营销进行总结与反思，以期促进我国网络广告的繁荣和良性发展。

关键词: 大数据, 网络广告, 精准营销

\section{1. 引言}

大数据时代的到来给社会带来了一股强劲的变革 力量, 通过对这些数据进行深入挖掘、再利用、重新组 合和扩展可以最大程度发挥其价值。身处大数据的技术 环境下, 我们的生活、工作以及学习等方方面面都发生 着或多或少的变革, 而网络广告作为其中的一部分, 也 正接受者大数据技术带来的经济效益回报。近几年以来, 我国的网络用户已经形成很大的规模, 逐渐从城市扩展 到农村, 影响面也越来越广泛, 网民的快速增长给网络 广告的精准营销提供了机会。根据艾瑞咨询数据, 2018 年度中国网络广告市场规模达到 4844.0 亿元, 同比增 长 $29.2 \%$ 。从绝对值来看, 中国网络广告产业生命力依 然旺盛, 预计在 2021 年市场规模将达到近万亿, 而大
数据等数字营销技术依旧是推动网络营销市场的强大 助燃剂。基于大数据的分析技术, 促使企业在日益竞争 激烈的市场环境中越来越以 “消费者为中心” 作为自己 的求生之本, 以此来不断满足消费者日渐高涨的美好生 活的需求。因此, 基于大数据的网络广告伴随着天时地 利人和的独特优势已经开始席卷中国。

伴随网络广告的兴盛, 以四大传统媒体为典型代表 的传统广告的市场开始急剧萎缩, 曾经强劲的核心竞争 力也在一步步地消退, 严重的生存危机接踵而至。在大 数据技术的作用下, 当今的广告产业正进行着各领域的 交汇、融合, 数字营销主导的广告, 基于大数据的驱动 技术, 正犹如雨后春笋般茁壮成长。在不断变化的动态 环境中, 传统网络广告如何根据大数据的技术, 实现用 户需求的个性化以及网络广告的高效化，提高网络广告 的高校性和精准性, 已经成为未来网络广告的重要研究 
(4) 研究网络精准广告, 注重精准广告的模式以及

方向。尽管数据挖掘分析技术会给网络广告营销带来华 丽变革, 但同时, 在应用过程中存在着不少问题亟待我 们采取对策, 例如大数据技术在我国发展并不成熟, 企 业并不能充分地利用大数据, 导致广告精准性不够; 大 数据广告人才紧缺; 用户安全隐私泄漏等等一系列的问 题都需要我们深思。另外在大数据技术支持的基础上, 如何根据实际情况, 制定相应的广告营销应用策略, 对 网络广告的效率有更上一层楼的作用。

因此, 为使网络广告市场蓬勃发展, 深入研究大数 据时代下网络广告精准营销策略具有十分重要的意义。

\section{2. 文献梳理}

\section{1. 大数据概念}

“大数据 (big data), 又称巨量资料, 是指所涉及 的资料量规模巨大到无法透过目前主流常见的软件工 具, 在合理时间内经过算取、管理、传递、分析等手段 帮助企业经营更积极目的的资讯” [1]。一般意义来说， 大数据是指在无可容忍的时间内用传统 IT 技术和软 硬件工具对其进行感知、获取、管理、处理和服务的数 据集合; 大数据是需要新处理模式才能具有更强的决策 力、洞察发现力和流程优化能力的海量、高增长率和多

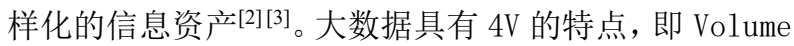
(数量大)、Variety (多样性)、Velocity (高频速度)、 Value (价值大)。同时, 大数据使得我们的生活更加数 据化, 海量的数据分析使得信息更加个人化, 决策更加 精准化 ${ }^{[4]}$ 。

\section{2. 大数据与网络广告}

随着大数据的快速发展, 越来越多的学者致力于研 究大数据与网络广告这一营销领域的有机结合。主要分 为以下四个方面:

(1) 网络广告形式和传播策略为着眼点, 研究大数 据对行业变革以及广告营销策略的影响。例如李亦宁和 杨琳（2014）从微观信息传播和宏观产业格局两个层面 论述大数据对广告产业的影响, 并对其未来发展趋势提 出期望 ${ }^{[5]}$ 。袁清 (2017) 提出在大数据影响下, 企业如 何改革其营销体系以增加企业的竞争力 ${ }^{[6]}$ 。

(2) 研究大数据 RTB 模式。肖鸿江和孙国华 (2013) 对 RTB 广告模式进行了详细的解读 ${ }^{[7]}$ 。许正林和杨瑶 （2015）详细分析了基于 RTB 的网络广告的市场运作 模式, 并 RTB 模式进行了更加系统的总结, 阐述现状, 发现不足，提出解决问题的策略并做了展望 ${ }^{[8]}$ 。

(3) 研究在大数据的影响下网络营销的策略。陈慧 和王明宇 (2014) 讲述了大数据网络营销的特征, 并制 定策略实现如何进行网络精准营销和如何实现大数据 ${ }^{[9]}$ 。 金金（2018）针对移动互联网, 分析了其存在的优势和 问题, 并为广告的精准性提供策略 ${ }^{[10]}$ 。
实际应用效果。例如刘英贵和李海峰 (2013) 通过优劣 势、发展的前景等方面对 cookie 跟踪以及跨平台微博 这两种营销方式进行深度分析, 探讨如何把握住消费者 的兴趣, 实现广告的精准营销模式 ${ }^{[11]}$ 。Xu 等 (2016) 引 入了知识融合分类法来理解传统营销分析、大数据分析 和新产品成功之间的关系, 认为提高新产品的知识融合 不是自动的, 需要通过战略选择来获得其效益 ${ }^{[12]}$ 。

\section{3. 大数据时代网络广告精准营销存在问题}

大数据技术已经开始在中国的网络广告市场中慢 慢得以应用, 并驱动了网络广告的效率和精准性, 但同 时也存在着一些阻碍其发展的问题。

首先，由于大数据技术的成熟度问题，不少网民认 为网络广告的精准度还不够。大数据的分析功能在一定 程度上并没有使网络广告的营销做到十分精准，无法实 现受众用户的精准洞察, 用户的个性需求并没有通过数 据技术充分体现出来。其次, 由于大数据技术正处于发 展阶段, 部分网民对此并不是十分了解。在汶览网页过 程中, 许多网民担忧自己的记录受到监视而侵害了他们 的个人隐私，并且伴随着一些强制性广告的产生，网民 对借助大数据进行网络广告营销具有一定的排斥心理, 无法完全接受。此外, 不少网络广告没有很好利用大数 据来加强网络广告精准营销的精准性, 缺乏创意, 无法 转化成有效的点击和阅读率。因此网络广告还具有十分 大的创意优化空间。最后, 数据和广告的关联深度还不

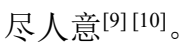

针对大数据时代网络广告出现的问题, 制定出一系 列的策略迫在眉睫, 以此来促进网络广告又好又快的发 展。

\section{3. 大数据时代网络广告精准营销策略}

针对上文表述的网络广告现状以及大数据目前在 网络广告中的应用, 此部分从技术基础和应用形式两个 层次, 以理论结合实际的方法就如何促进网络广告精准 营销提出一些看法和建议, 希望能为我国网络广告的发 展尽绵薄之力。

\section{1. 增强大数据技术成熟度}

马云曾说过: “假如我们有了一个数据预报台, 就像 为企业装上了一个 GPS 和雷达, 企业的出海将会更有 把握。”大数据时代, 数据便是网络广告进行精准营销 的必备武器, 对这把进行不停地锻造, 提高广告对数据 的挖掘、收集、整理以及分析能力, 促进广告内容与用 户需求的贴合程度 ${ }^{[13]}$ 。 


\section{3. 瞄准移动互联网广告平台, 开展关系营 销}

目前, 我国的移动互联网广告平台在百度、阿里巴 巴、腾讯、新浪微博等互联网巨头的冲击下正在形成更 加细化的行业布局。通过广告平台这一中介, 企业可以 对用户的兴趣、需求进行追踪分析, 并通过其所处圈子 的挖掘, 可以更为精准找到目标群体。社交网络的数据 具有更高的活性、真实性和动态性。企业可以通过大数 据技术根据用户的互动情况挖掘、分析用户的人际脉络, 开展关系营销。小米的微博关系营销就是一个很好的例 子。

\section{4. 精准广告形式，利用数据优化广告创意}

\section{4. 1, 根据用户所处情境推送广告}

伴随 GPS 定位系统、LBS 等技术的不断完善和成 熟, 企业通过对位置、时间、行为等数据进行智能分析, 并借助数据实时追踪预测等技术, 可以对用户现在甚至 之后的所处情境进行捕捉并有效识别, 采取合适的广告 形式, 避免广告内容和用户所处情境的不匹配。比如企 业可以根据消费者 “下班后”、“在逛街”、“睡觉前” 等不同的情境, 推送用户会感兴趣的广告内容, 很有可 能会使广告效果事半功倍 ${ }^{[15]}$ 。

互联网每时每分每秒都在产生超大量的数据, 但是 并不是所有的数据都是有用的。企业要学会对信息去噪, 将一些无关的信息及时剔除出去, 并甄别数据的重要程 度进行深度分析, 这对大数据技术又提出了很高的要求。 在筛选出有用数据以后, 要建立起科学的预测系统, 精 准定位用户需求, 以提供更加优质的服务。亚马逊等许 多电商平台根据用户的特征、购物车以及搜索历史等对 用户的偏好和需求进行分析，以此投放更加精准的广告。

\section{2. 保护用户隐私, 提升用户体验}

通过保护用户的隐私, 能够有效降低广大网名对大 数据技术的排斥和减轻用户对数据泄漏形成的恐慌, 从 而提高用户在使用网络的体验, 促进大数据和互联网的 双重发展。为了做到这一点, 首先, 以立法的形式明确 用户隐私范畴, 建立起保护隐私的相关法律法规; 其次, 用户要提高自我隐私的防范意识。接着, 政府要加强数 据市场的管理力度, 定期抽查数据市场相关行业实行情 况, 及早发现存在的不规范行为, 做好履行监管的职责; 最后, 企业要提高行业自我规范。数据公司有责任要加 强其职业道德操守, 严格增强其行业的自我规范 ${ }^{[14]}$ 。

\subsection{2, 丰富广告创意, 增加感染力}

数据、技术是实现精准广告很重要的一部分, 但并 不是全部。为防止广告显得生硬而处于被动地位, 广告 主应充分调动其主观能动性, 尽量以故事性和新颖性的 内容为依托, 完善与用户的交互功能, 促进广告美轮美 负的视觉体验避, 免用户因不感兴趣而把广告遗忘在角 落 ${ }^{[13]}$ 。谷歌在设计巴宝莉口红的网页广告时, 将用户体 验和数据技术结合, 当用户在屏幕上捕捉亲吻和传递亲 吻时，会伴随优美的音乐，进而达到良好的交互效果。

\section{5. 深度挖掘数据信息, 加强关联广告}

广告企业目前正面临着 “数据海量，信息缺乏” 这 但是也不可忽视相关关系。关联广告的挖掘也是进行网 络广告精准营销的重要一步[14]。在 1990 的《纸牌屋》 电影翻牌过程前, Netflix 公司在其公司数据库了保存 了 3000 万用户的收视选择、 400 万条评论以及 300 万 次主题搜索。“数据决定拍摄” 这一举动, 使这部影片 取得了非同凡响的收视率。显然, 这对网络广告的精准 营销也有着很好的借鉴意义，比如不同数据之间也可以 进行参照对比, 寻找数据之间有可能存在的规律, 实现 个巨大的难题。在大数据时代, 因果关系关系固然重要, 
[5] Li, Y. N ., Yang, L. (2014) Evolution and reconstruction of advertising industry ecology under the background of big data. Research on Advertisement, 2: 86-88.

[6] Yuan, Q. (2016) The reconstruction and development of marketing system in the era of big data. Journal of Commercial Economics, 24: 46-48.

[7] Xiao, H. J., Sun, G. H. (2013) Design of Internet advertising process based on real-time bidding. Technology Information, 37: 130-132.

[8] Xu, Z. L ., Yang, Y. (2105) Research and analysis of mobile Internet RTB advertising precise delivery model and its marketing strategy based on big data. Journal of Shanghai University (Social Sciences), 32: 104-117.

[9] Chen, H., Wang, M. Y. (2014) Big data: making Internet marketing more accurate. E-business Journal, 7: 32-33.

[10] Jin, J. (2018) Mobile Internet advertising precision marketing strategy in the era of big data. Modern economic information, 1: 323-24.

[11] Liu, Y. G., Hai, H. F. (2013) Research on the marketing method of precise advertisement in new media communication. Research on Advertisement, 4: 86-88.

[12] Xu, Z., Frankwick, G. L., Ramirez, E. (2016) Effects of big data analytics and traditional marketing analytics on new product success: A knowledge fusion perspective. Journal of Business Research, 69: 15621566.

[13] Zou, T. (2015) Research on the application of big data in online advertising. Hunan: Xiangtan University.

[14] Wu, W. (2016) Research on precision marketing of mobile Internet advertising in the era of big data. Chongqing: Southwest University.

[15] Zhang, X. M. (2017) Research on the innovation of precision marketing of network media advertising under the background of big data. Contemporary Economics, 4: 86-88.
[3] Meng, X. F., Ci, X. (2013) Big data management: concepts, techniques and challenges. Journal of Computer Research and Development, 50: 146-169.

[4] Mcafee, A., Brynjolfsson, E. (2012) Big data: the management revolution. Harvard business review, 90: 6-60. 